\title{
Logistic Model Tree Classifier for Condition Monitoring of Wind Turbine Blades
}

\author{
A. Joshuva, G. Deenadayalan, S. Sivakumar, \\ R. Sathishkumar, R. Vishnuvardhan
}

\begin{abstract}
Wind energy is one of the essential renewable energy resources because of its consistency due to the development of the technology and relative cost affordability. The wind energy is converted into electrical energy using rotating blades which are connected to the generator. Due to environmental conditions and large construction, the blades are subjected to various faults and cause the lack of productivity. The downtime can be reduced when they are diagnosed periodically using condition monitoring technique. These are considered as a machine learning problem which consists of three phases, namely feature extraction, feature selection and fault classification. In this study, statistical features are extracted from vibration signals, feature selection are carried out using $\mathrm{J48}$ algorithm and the fault classification was carried out using logistic model tree algorithm.
\end{abstract}

Keywords--- Fault Diagnosis; Condition Monitoring; Statistical Features; J48 Algorithm; Logistic Model Tree (LMT) Algorithm.

\section{INTRODUCTION}

In recent years, wind energy becomes the most demanded resource in power generation. Because of worldwide ecological contamination due to other resources, the sustainable energy and natural resources like wind energy have risen [1]. "Wind energy is one of the efficient renewable sources and an alternative option for commonly used sources. The objective of the wind turbine is to boost ideals by extreme energy extraction and in this manner, the performance of the wind turbine frequently differs with wavering winds. To make wind energy more focused from different sources of energy, like performance, accessibility, reliability, effectiveness, the life of turbines must be improved [2].

Generally, horizontal axis wind turbine (HAWT) is used for power generation since it is more effective than the vertical axis wind turbine [3]. Most HAWT are with three rotor blades typically placed upwind of the tower and the nacelle. The nacelle is typically furnished with anemometers and a wind vane to calculate the wind rate and direction of the wind. The nacelle also contains aviation light signal and

\footnotetext{
Manuscript received September 16, 2019.
}

A. Joshuva*, Centre for Automation and Robotics (ANRO), Department of Mechanical Engineering, Hindustan Institute of Technology and Science, Old Mahabalipuram Road, Padur, Kelambakam, Chennai, T.N, India. (e-mail: joshuva1991@gmail.com)

G. Deenadayalan, Centre for Automation and Robotics (ANRO), Department of Mechanical Engineering, Hindustan Institute of Technology and Science, Old Mahabalipuram Road, Padur, Kelambakam, Chennai, T.N, India.

S. Sivakumar, Department of Mechanical Engineering, Hindustan Institute of Technology and Science, Old Mahabalipuram Road, Padur, Kelambakam, Chennai, T.N, India.

R. Sathishkumar, Department of Automobile Engineering, Hindustan Institute of Technology and Science, Old Mahabalipuram Road, Padur, Krishna College of Engineering and Technology, Coimbatore, T.N, India. Kelambakam, Chennai, T.N, India.

R. Vishnuvardhan, Department of Mechatronics Engineering, Sri

the key segments of the wind turbine like gearbox, mechanical brake, electrical generator, control frameworks, yaw drive, and so forth [4].

The main objective of condition monitoring method is to create the power yield as calculated. When there is an awareness of the time and categories of defects, maintenance activities can be arranged ahead of time [5].

Thus, condition monitoring of blade fault is a critical activity. There are two types of approaches which are carried out for condition monitoring: traditional approach and machine learning approach. The traditional approach is mainly used for frequency component does not change with respect to time.

Rotating machines produce non-stationary signals. Since the frequency components change due to wear and tear, fault discrimination is very difficult using an automated system in the traditional approach. Hence, not preferred. In machine learning approach, algorithms have the capability to learn continuously and adapt themselves to the varying situations. Researchers often resort to machine learning approach for fault diagnosis of mechanical systems."

Many studies were carried out using machine learning studies and simulation studies to name a few, Godwin and Peter Matthews [6] have done classification and detection of wind turbine pitch faults through SCADA data analysis and RIPPER algorithm which yield them $87.05 \%$ classification accuracy in pitch angle fault. "N. Dervilis et al., [7] carried out a research on damage diagnosis for a wind turbine blade using pattern recognition such as principal component analysis (PCA), nonlinear principal component analysis (NLPCA), artificial neural network (ANN), auto-associative neural network (AANN) and Radial Basis Functions (RBF) using vibration signals.

Mark Mollineaux et al., [8] carried out a work on damage detection methods of wind turbine blade testing with wired and wireless accelerometer sensors using benchmark data and autoregressive moving average (ARMA) and Continuous Wavelet Transform (CWT) used as modeling techniques.

Mahmood Shafiee et. al [9] have done work on an opportunistic condition based maintenance policy for offshore wind turbine blades subjected to stress corrosion cracking and environmental shocks and simulated using MATLAB.

H. M. Slot et al., [10] have made a review of coating life models on the leading edge erosion of coated wind turbine blades and he suggested the material and prediction of the lifetime of the blade.

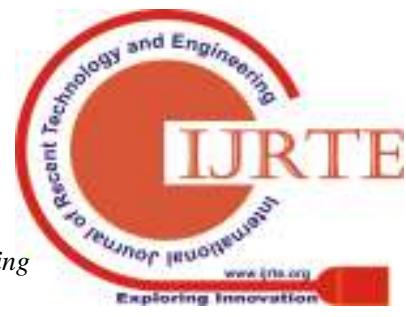


Shizhong Zhang et al., [11] carried out a work on design and analysis of jet based laboratory equipment for performance evaluation on the erosion of wind turbine blade coatings.

Xiang Li et al., [12] have carried out a research on crack growth, sparse pursuit for wind turbine blade using vibration data and lamb wave propagation. Wei Xie et al., [13] have made a novel folding blade of wind turbine rotor for effective power control using MATLAB and achieved maximum reduction of $51.1 \%$ in pitch control (Optimum fold angles of 550 and 900).

Simon Hoell and Piotr Omenzetter [14] have carried out a structural damage detection in wind turbine blades based on time series representations of dynamic responses using vibration data and cross-correlations, principal component analysis (PCA), genetic programming (GP) as the diagnostic algorithms."

Wang Yongzhi et al., [15] have carried out a study on a composite wind turbine blade aerodynamic and structurally integrated design optimization based on radial basis function (RBF) meta-model, blade element momentum (BEM) theory, finite element method (FEM) and multi-island genetic algorithm (MIGA).

"This paper aims to reduce the mass of blade under some constraints, including the power and deflection at the rated wind speed, and the strength and deflection under the ultimate case. P.U. Haselbach et al., [16] studied the effect of delamination on local buckling in wind turbine blades using FEM analysis.

They simulated by bending the blade in flap-wise direction and conducted the experiment. Although numerous works were carried out using simulation studies, only a very few of the experimental studies for condition monitoring on

Some researchers carried out fault diagnosis system using machine learning approach and they considered very few faults in their study.

Only selected algorithms were used to perform the fault prediction on wind turbine blades and many algorithms are yet to be studied [17]. Figure 1 shows the methodology of the work done."

The contribution of the present study

1. This study considers more than two faults for fault diagnosis of the wind turbine (blade crack, erosion, hub-blade loose connection, pitch angle twist and blade bend).

2. Vibration signals are taken for the blade using data acquisition.

3. Statistical analysis is used for feature extraction.

4. The $\mathbf{J} 48$ algorithm is used for feature selection.

5. Logistic model tree (LMT) algorithm is used for feature classification.

The rest of the paper is organized as follows. Section 2 presents the experimental setup and experimental procedure.

In section 3, feature extraction is explained, followed by feature selection in section 4 . The classifiers used in this study are explained in section 5 . The classification accuracy of the LMT model was discussed in section 6. Conclusions are presented in the final section (section 7). wind turbine blades were carried out.

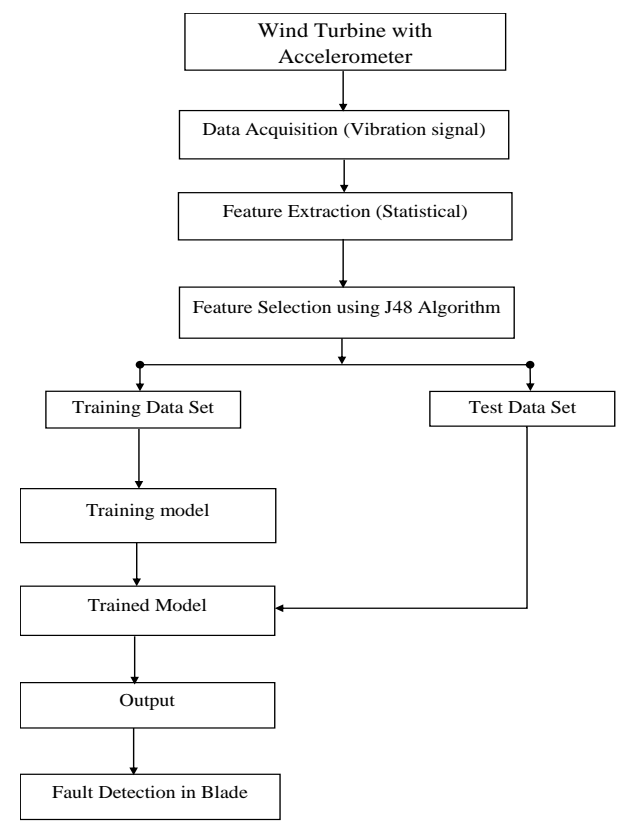

Figure 1: Methodology

\section{EXPERIMENTAL STUDIES}

The main aim of this study is to classify whether the blades are in good condition or in a defective state. "If it is defective, then the objective is to identify the type of fault. The experimental setup and experimental procedure are described in the following subsections.

\section{Experimental Setup}

The experiment was carried out on a $50 \mathrm{~W}, 12 \mathrm{~V}$ variable speed wind turbine (MX-POWER, model: FP-50W-12V) [18]. The technical parameters of a wind turbine are given in Table 1. The wind turbine was mounted on a fixed steel stand in front of the open circuit wind tunnel outlet. The wind tunnel speed ranges from $5 \mathrm{~m} / \mathrm{s}$ to $15 \mathrm{~m} / \mathrm{s}$ and acts as a wind source to start the wind turbine. The wind speed was varied continuously in order to simulate the environmental wind condition. The experimental setup is shown in Figure 2. Piezoelectric type accelerometer was used as a transducer for acquiring vibration signals. It has high sensitivity for detecting faults. Hence, accelerometers are widely used in condition monitoring. In this case, an uniaxial accelerometer of $500 \mathrm{~g}$ range, $100 \mathrm{mV} / \mathrm{g}$ sensitivity, and resonant frequency around $40 \mathrm{~Hz}$ was used.

Table 1: Technical Parameters of Wind Turbine

\begin{tabular}{|l|l|}
\hline Model & FP-50W-12V \\
\hline Rated Power & $50 \mathrm{~W}$ \\
\hline Rated Voltage & $12 \mathrm{~V}$ \\
\hline Maximum Current & $4 \mathrm{~A}$ \\
\hline Rated Rotating Rate & $850 \mathrm{rpm}$ \\
\hline Start-up Wind Speed & $2.5 \mathrm{~m} / \mathrm{s}$ \\
\hline Cut-in Wind Speed & $3.5 \mathrm{~m} / \mathrm{s}$ \\
\hline Cut-out Wind Speed & $15 \mathrm{~m} / \mathrm{s}$ \\
\hline Security Wind Speed & $40 \mathrm{~m} / \mathrm{s}$ \\
\hline Rated Wind Speed & $12.5 \mathrm{~m} / \mathrm{s}$ \\
\hline Engine & Three-phase permanent magnet generator \\
\hline Rotor Diameter & $1050 \mathrm{~mm}$ \\
\hline Blade Material & Carbon fiber reinforced plastics \\
\hline
\end{tabular}


The piezoelectric accelerometer (DYTRAN 3055B1) was mounted on the nacelle near to the wind turbine hub to record the vibration signals using an adhesive mounting technique. It was connected to the DAQ system through a cable. The data acquisition system (DAQ) used was NI USB 4432 model. The DAQ card has five analogue input channels with a sampling rate of 102.4-kilo samples per second with 24-bit resolution. The accelerometer is coupled to a signal conditioning unit which consists of an inbuilt charge amplifier and an analogue-to-digital converter (ADC). From the ADC, the vibration signal was taken. These vibration signals were used to extract features through feature extraction technique. One end of the cable is plugged to the accelerometer and the other end to the AIO port of DAQ system. NI - LabVIEW was used to interface the transducer signal and the system (PC).

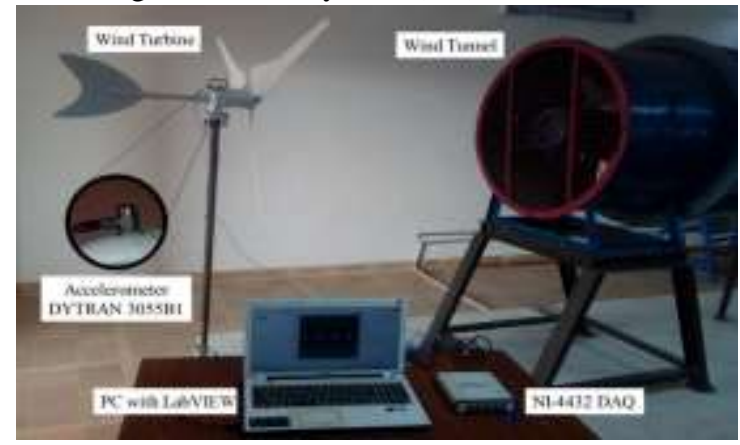

Figure 2: Wind Turbine Setup

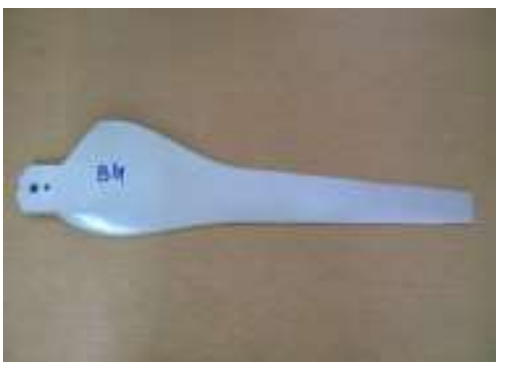

Good condition blade

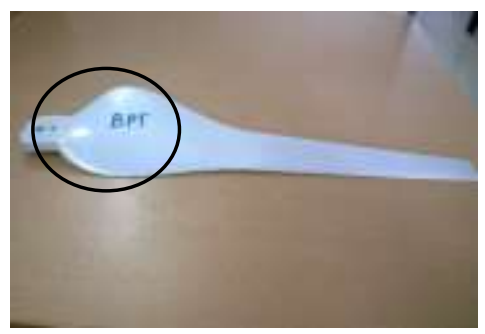

Blade with pitch angle twist

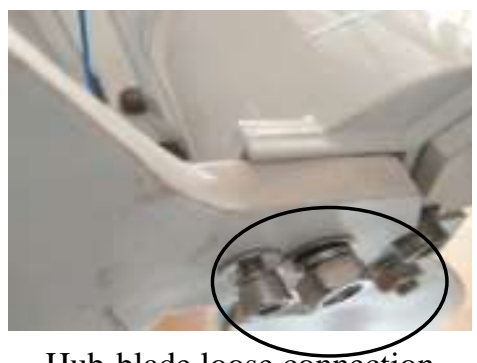

Hub-blade loose connection

\section{Experimental Procedure}

In the present study, three-blade variable horizontal axis wind turbine (HAWT) was used. Initially, the wind turbine was considered to be in good condition (free from defects, new setup) and the signals were recorded using an accelerometer. These signals were recorded with the following specifications.

1. Sample length: The sample length was chosen long enough to ensure data consistency; and also the following points were considered. ARMA measures are more meaningful when the number of samples is sufficiently large. On the other hand, as the number of samples increases the computation time increases. To strike a balance, a sample length of 10000 was chosen.

2. Sampling Frequency: The sampling frequency should be at least twice the highest frequency contained in the signal as per Nyquist sampling theorem. By using this theorem sampling frequency was calculated as $12 \mathrm{kHz}(12000 \mathrm{~Hz})$.

3. Number of samples: Minimum of 100 (hundred) samples were taken for each condition of the wind turbine blade and the vibration signals were stored in data files.

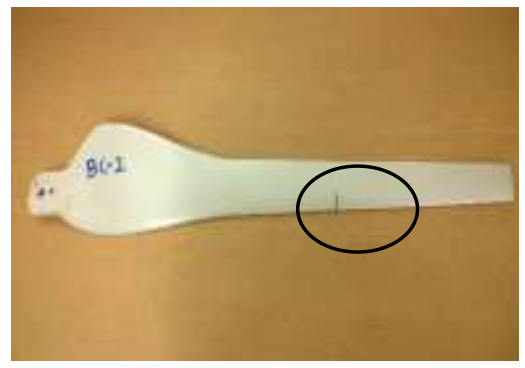

Blade with crack

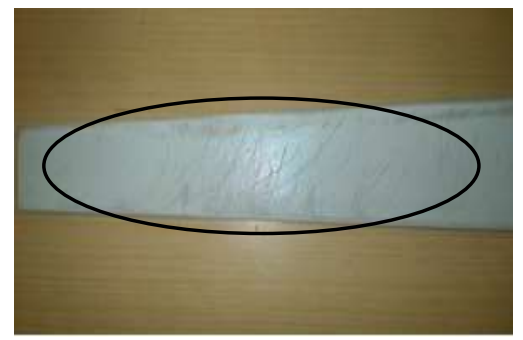

Blade with erosion

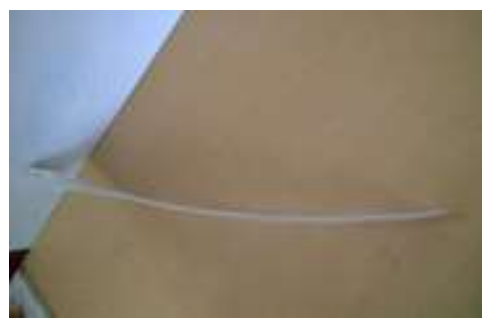

Blade with bend (Top View)

Figure 3: Various Blade Fault Conditions

The following faults were simulated one at a time while all other components remain in good condition and the 
corresponding vibration signals were acquired. Figure 3 shows the different blade fault conditions which are simulated on the blade.

a) Blade bend (BB): This fault occurs due to the high-speed wind and complex forces caused by the
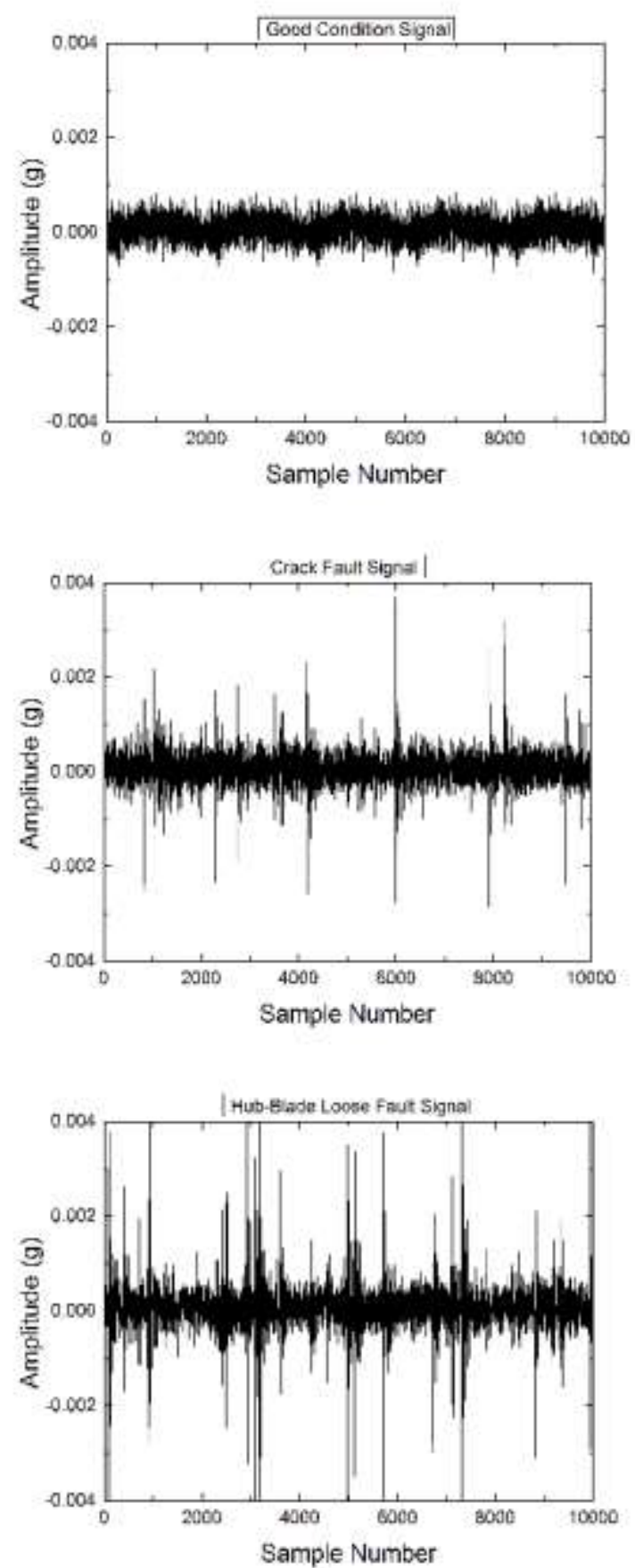

wind. The blade was made to flap wise bend with $10^{\circ}$ angle.

b) Blade crack (BC-2): This occurs due to foreign object damage on the blade while it is in operating condition. On the blade, $15 \mathrm{~mm}$ crack was made.
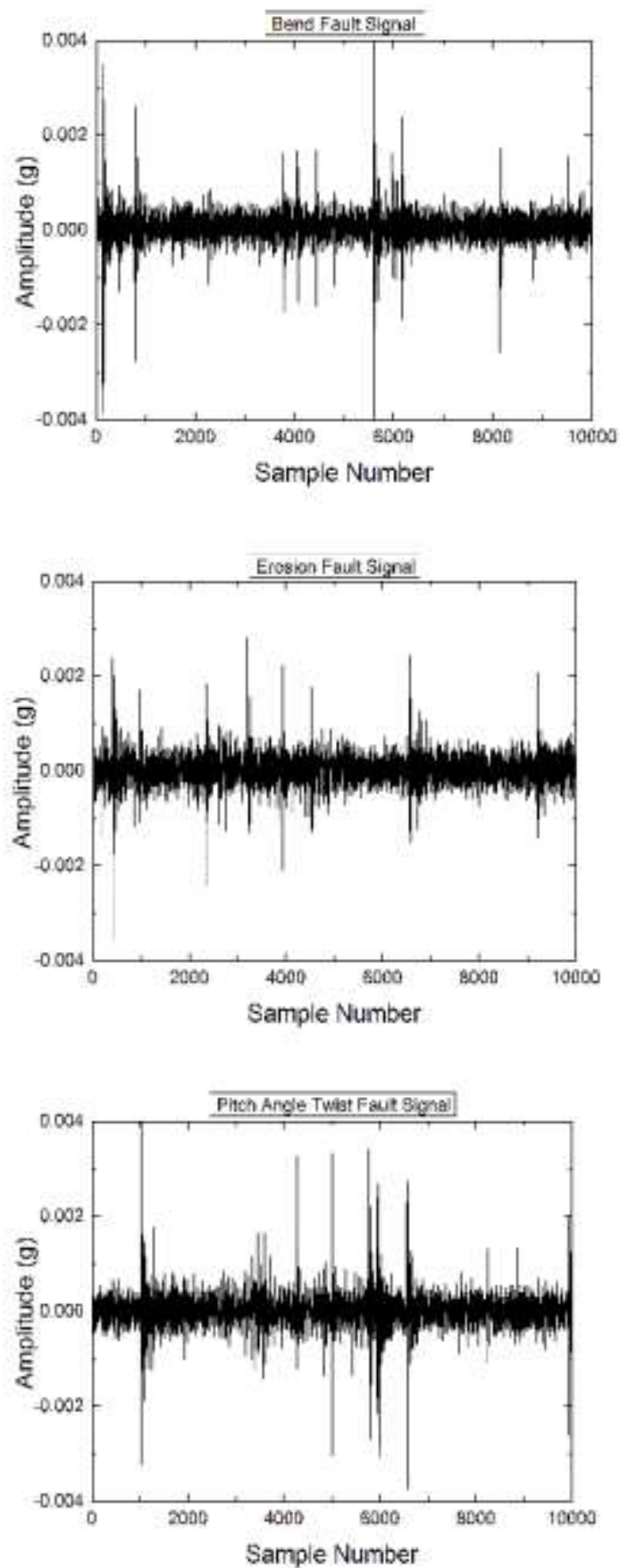

Figure 4: Vibration Signal Plot

c) Blade erosion (BE): This fault is due to the erosion of the top layer of the blade by the highspeed wind. The smooth surface of the blade was eroded using emery sheet $(320 \mathrm{Cw})$ to provide an erosion effect on the blade.

d) Hub-blade loose contact: This fault generally occurs on a wind turbine blade due to an excessive runtime or usage time. The bolt connecting the hub and blade was made loose to obtain this fault.

e) Blade pitch angle twist (PAT): This fault occurs due to the stress on the blade caused by high-speed wind. This makes the pitch get twisted, creating a heavy vibration to the framework. To attain this fault, blade pitch was twisted about $12^{\circ}$ angle with respect to the normal blade condition.

In Figure 4, the vibration signals (sample number vs. amplitude) are shown which were taken for different conditions of the wind turbine blade (good condition blade, blade bend, blade erosion, hub-blade loose connection, blade crack and pitch angle twist). 


\section{FEATURE EXTRACTION - STATISTICAL ANALYSIS}

The vibration signals were acquired for good and other faulty conditions of the blade. If the time domain sampled signals are given directly as inputs to a classifier, then the number of samples should be constant. "The number of samples obtained which are the functions of rotation of the blade speed. Hence it varies with speed. Also, the number of digitized data points in a signal is too large; generally the work of classifiers may not handle it efficiently. Therefore, a few features must be extracted before the classification process. Descriptive statistical parameters [19] such as sum, mean, median, mode, minimum, maximum, range, Skewness, kurtosis, standard error, standard deviation and sample variance were computed to aid as features in the feature extraction process.

\section{FEATURE SELECTION - J48 ALGORITHM}

$\mathrm{J} 48$ decision tree algorithm is adapted from the $\mathrm{C} 4.5$ algorithm in WEKA [20]. "It consists of a number of branches, one root, a number of nodes, and a number of leaves. One branch is a chain of nodes from the root to a leaf, and each node involves one attribute. The occurrence of an attribute in a tree provides information about the importance of the associated attribute [21]. A decision tree is a tree based knowledge representation methodology used to represent classification rules. J48 decision tree algorithm is a widely used one to construct decision trees [22]. The procedure of forming the decision tree and exploiting the same for feature selection is characterized by the following:

1. The set of features available at hand forms the input to the algorithm; the output is the decision tree.

2. The decision tree has leaf nodes, which represent class labels, and other nodes associated with the classes being classified.

3. The branches of the tree represent each possible value of the feature node from which they originate.

4. The decision tree can be used to classify feature vectors by starting at the root of the tree and moving through it until a leaf node, which provides a classification of the instance, is identified.

5. At each decision node in the decision tree, one can select the most useful feature for classification using appropriate estimation criteria. The criterion used to identify the best feature invokes the concepts of entropy reduction and information gain.

Information gain measures how well a given attribute separates the training examples according to their target classification. The measure is used to select the candidate features at each step while growing the tree [23]. Information gain is the expected reduction in entropy caused by portioning the samples according to this feature.

Information gain $(S, A)$ of a feature $A$ relative to a collection of examples $S$, is defined as:

$$
\operatorname{Gain}(S, A)=\operatorname{Entropy}(S)-\sum_{v \in \operatorname{Value}(A)} \times \frac{\left|S_{v}\right|}{|s|} \operatorname{Entropy}\left(S_{v}\right)
$$

where Value $(A)$ is the set of all possible values for attribute $A$, and $S_{v}$ is the subset of $S$ for which feature $A$ has value $v$. Note the first term in the equation for gain is just the entropy of the original collection $S$ and the second term is the expected value of the entropy after $S$ is partitioned using feature $A$. The expected entropy described by the second term is simply the sum of the entropies of each subset $S_{v}$, weighted by the fraction of samples $\left|S_{v}\right| /|\mathrm{S}|$ that belong to $S_{v}$. Gain $(S, A)$ is, therefore, the expected reduction in entropy caused by knowing the value of feature $A$. Entropy is a measure of homogeneity of the set of examples and it is given by

$$
\operatorname{Entropy}(S)=\sum_{i-1}^{e}-P_{i} \log _{2} P_{i}
$$

where, $c$ is the number of classes, $P_{i}$ is the proportion of $S$ belonging to class ' $i$ '.

The $\mathrm{J} 48$ decision tree algorithm has been applied to the problem of feature selection. The input to the algorithm is the set of statistical features described above and output of the decision tree shown in Figure 5. It is clearly shown that the top node is the best node for classification. The other features in the nodes of decision tree perform in descending order of significance. It is to be mentioned here that only features that contribute to the classification appear in the decision tree and other features do not contribute much. The features which have the less discriminating capability can be consciously discarded by deciding on the threshold. This concept is made use for selecting good features. The algorithm identifies the good features for the purpose of classification of the given training data set, and thus reduces the domain knowledge required to select good features for pattern classification problem [24-26]. Referring from Figure 5, one can identify the most dominating features to represent the blade conditions are the sum, range, standard deviation, and kurtosis.

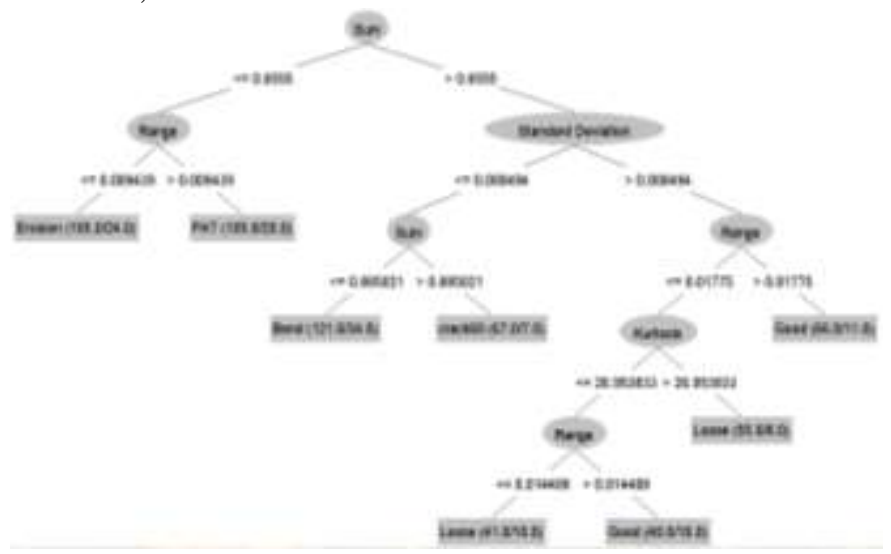

Figure 5: J48 Tree Classification for Feature Selection

\section{FEATURE CLASSIFICATION - LOGISTIC MODEL TREE (LMT) ALGORITHM}

A logistic model tree essentially comprises a normal decision tree arrangement with logistic regression tasks at the leaves [27]. As in normal decision trees, an assessment on one of the qualities is connected with each internal hub. For an identified property with $k$ values, the hub has $k$ child hubs, and illustrations are sorted down one of the $k$ branches relying upon their estimation of the feature. For numeric features, the hub has two child hubs and the test comprises 
of contrasting the characteristic significance to the threshold. Generally, a logistic model tree comprises of a tree arrangement that is comprised of an arrangement of internal or non-terminal hubs $N$ and an arrangement of leaves or terminal hubs $T$. Let $S$ indicate the entire occurrence in space, spread over by all characteristics that are available in the information [28]. At that point, the tree structure gives a separate section of $S$ into areas $S_{t}$, and each area is characterized by a leaf in the tree.

$$
S=\bigcup_{t \in T} S_{t^{\prime}} \text { St } \cap S t^{\prime}=\emptyset \text { for } t \neq t^{\prime}
$$

Not like all decision trees, the leaves $t \in T$ has a related logistic regression function $f_{t}$ rather than only a class name. The regression function $f_{t}$ considers a subset $V_{t} \subseteq V$ of all characteristics existing in the data and models the class relationship possibilities as

$$
\operatorname{Pr}(G=j \mid X=x)=\frac{e^{F_{j}(x)}}{\sum_{k=1}^{j} e^{F_{k}(x)}}
$$

$$
\text { Where } \quad F_{j}(x)=\alpha_{0}^{j}+\sum_{v \in v_{t}} \propto_{v}^{j} \cdot v
$$

If $\alpha_{v k}^{j}=0$ for $v_{k} \neq V_{t}$. The model denoted by the whole logistic model tree is given by,

$$
f(x)=\sum_{t \in T} f_{t}(x) \cdot I\left(x \in S_{t}\right)
$$

Where $I\left(x \in S_{t}\right)$ is 1 if $x \in S_{t}$ and 0 otherwise.

\section{RESULTS AND DISCUSSION}

The vibration signals were acquired for good condition and faulty blade conditions using DAQ (total 600 samples were collected; 100 samples for each condition). From vibration signals, twelve descriptive statistical features were extracted. Out of them, four best contributing features were selected using J48 decision tree algorithm. They are the sum, range, standard deviation, and kurtosis. From Figure 5, the feature 'sum' is the most contributing features when compared to other features. The other contributing features are a range, standard deviation, and kurtosis.

The minimum number of instances per leaf and the number of data used for reduced-error pruning was kept at 50 for selecting 4 dominating feature in $\mathrm{J} 48$ decision tree algorithm. The rest of the features like mean, median, mode, minimum, maximum, skewness, sample variance and standard error were eliminated as they contribute very less in fault classification. With the selected features, the fault classification process was carried out using logistic model tree. The classification accuracy of LMT algorithm using WEKA software package was found to be $90.33 \%$ with the minimum number of instances to be 20 and the logitboost iteration to be -2 . The confusion matrix of the logistic model tree algorithm is shown in Table 2 . In confusion matrix, the diagonal elements represent the correctly classified instances and the others are misclassified instances [29]. Also one can observe more misclassifications between good and loose conditions. For the loose condition, the bolts between the hub and the blade were made loose (please note that the blade was in good condition). However, at high wind speed, the blade can stick to the hub and behave like a good condition during operation. Because of this, the signature of the loose condition sometimes resembles good condition and the classifier finds difficult to distinguish between them; hence, more misclassifications.
From logistic model tree (LMT), the kappa statistic was found to be 0.884 . It is used to measure the arrangement of likelihood with the true class. The mean absolute error was found to be 0.0496 . It is a measure used to measure how close forecasts or prediction are with the ultimate result [30]. The root mean square error was found to be 0.1621 . It is a quadratic scoring rule which processes the average size of the error. The detailed class wise accuracy is shown in Table 3. The class wise accuracy is expressed in terms of the true positive rate (TP), false positive rate (FP), precision, recall, F-Measure, receiver operating characteristics (ROC) area [31-34].

TP is used to predict the ratio of positives which are correctly classified as faults. FP is commonly described as a false alarm in which the result that shows a given fault condition has been achieved when it really has not been achieved. The true positive (TP) rate should be close to 1 and the false positive (FP) rate should be close to 0 for a better classifier [35-41]. One can observe from table 3, the TP rate of most of the classes are close to 1 and FP rate were close to 0 . This reassures that the result presented by confusion matrix in Table 2.

Table 2: Confusion Matrix of LMT

\begin{tabular}{|l|l|l|l|l|l|l|}
\hline $\begin{array}{l}\text { Blade } \\
\text { condition } \\
\text { s }\end{array}$ & $\begin{array}{l}\text { Goo } \\
\mathrm{d}\end{array}$ & $\begin{array}{l}\text { Ben } \\
\mathrm{d}\end{array}$ & $\begin{array}{l}\text { Crac } \\
\mathrm{k}\end{array}$ & $\begin{array}{l}\text { Loos } \\
\mathrm{e}\end{array}$ & $\begin{array}{l}\text { Pitc } \\
\mathrm{h} \\
\text { twist }\end{array}$ & $\begin{array}{l}\text { Erosio } \\
\mathrm{n}\end{array}$ \\
\hline Good & 86 & 0 & 0 & 14 & 0 & 0 \\
\hline Bend & 0 & 92 & 4 & 0 & 0 & 4 \\
\hline Crack & 0 & 4 & 92 & 3 & 0 & 1 \\
\hline Loose & 13 & 0 & 4 & 82 & 1 & 0 \\
\hline $\begin{array}{l}\text { Pitch } \\
\text { twist }\end{array}$ & 0 & 0 & 0 & 0 & 95 & 5 \\
\hline Erosion & 0 & 3 & 0 & 0 & 5 & 92 \\
\hline
\end{tabular}

\begin{tabular}{|l|l|l|l|l|l|l|}
\hline \multicolumn{7}{|c|}{ Table 3: Class Wise Accuracy of LMT } \\
\hline Class & $\begin{array}{l}\text { TP } \\
\text { Rat } \\
\mathrm{e}\end{array}$ & $\begin{array}{l}\text { FP } \\
\text { Rate }\end{array}$ & $\begin{array}{l}\text { Precisio } \\
\mathrm{n}\end{array}$ & $\begin{array}{l}\text { Recal } \\
1\end{array}$ & $\begin{array}{l}\text { F- } \\
\text { Measur } \\
\mathrm{e}\end{array}$ & $\begin{array}{l}\text { ROC } \\
\text { area }\end{array}$ \\
\hline Good & $\begin{array}{l}0.8 \\
6\end{array}$ & $\begin{array}{l}0.02 \\
4\end{array}$ & 0.878 & 0.86 & 0.869 & $\begin{array}{l}0.97 \\
4\end{array}$ \\
\hline Bend & $\begin{array}{l}0.9 \\
3\end{array}$ & $\begin{array}{l}0.01 \\
4\end{array}$ & 0.93 & 0.93 & 0.93 & $\begin{array}{l}0.99 \\
4\end{array}$ \\
\hline Crack & $\begin{array}{l}0.9 \\
1\end{array}$ & 0.01 & 0.948 & 0.91 & 0.929 & $\begin{array}{l}0.98 \\
7\end{array}$ \\
\hline Loose & $\begin{array}{l}0.8 \\
5\end{array}$ & 0.04 & 0.81 & 0.85 & 0.829 & $\begin{array}{l}0.97 \\
1\end{array}$ \\
\hline $\begin{array}{l}\text { Pitch } \\
\text { twist }\end{array}$ & $\begin{array}{l}0.9 \\
4\end{array}$ & 0.01 & 0.949 & 0.94 & 0.945 & $\begin{array}{l}0.98 \\
8\end{array}$ \\
\hline $\begin{array}{l}\text { Erosio } \\
\mathrm{n}\end{array}$ & $\begin{array}{l}0.9 \\
3\end{array}$ & $\begin{array}{l}0.01 \\
8\end{array}$ & 0.912 & 0.93 & 0.921 & $\begin{array}{l}0.98 \\
4\end{array}$ \\
\hline
\end{tabular}

From 600 samples, 542 samples are correctly classified $(90.33 \%)$ and remaining 58 are misclassified $(9.67 \%)$. The time taken to build the model is about 0.23 seconds. This can be used in real time for the condition monitoring of the wind turbine due to low computational time.

The logistic model tree algorithm is a better classifier that can be used in multiclass fault prediction on wind turbine blades." 


\section{CONCLUSION}

The wind turbine is very important in the production of wind energy in the day to day life. This paper presents an algorithmic based clarification of vibration signals for the assessment of wind turbine blade conditions. From the acquired vibration data, a model was developed using data modeling technique. LMT algorithm was used to learn and classify the different conditions of the blade. The model is tested under 10-fold cross validation and correctly classified instances were found to be $90.33 \%$. The error rate is relatively less and may be considered for the blade fault diagnosis. Hence the logistic model tree can be practically used for the condition monitoring of wind turbine blade to reduce the downtime and to provide uninterrupted wind energy.

\section{REFERENCES}

1. Bellarmine GT, Urquhart J. Wind energy for the 1990s and beyond. Energy Conversion and Management. 1996 Dec 1; 37(12):1741-1752.

2. Márquez FP, Tobias AM, Pérez JM, Papaelias M. Condition monitoring of wind turbines: Techniques and methods. Renewable Energy. 2012 Oct 1; 46:169-178.

3. Veers PS, Ashwill TD, Sutherland HJ, Laird DL, Lobitz DW, Griffin DA, Mandell JF, Musial WD, Jackson K, Zuteck M, Miravete A. Trends in the design, manufacture and evaluation of wind turbine blades. Wind Energy: An International Journal for Progress and Applications in Wind Power Conversion Technology. 2003 Jul; 6(3):245-259.

4. Liu WY. The vibration analysis of wind turbine bladecabin-tower coupling system. Engineering structures. 2013 Nov 1; 56:954-957.

5. Ciang CC, Lee JR, Bang HJ. Structural health monitoring for a wind turbine system: a review of damage detection methods. Measurement science and technology. 2008 Oct 13; 19(12):122001. wind turbine pitch faults through SCADA data analysis. IJPHM Special Issue on Wind Turbine PHM. 2013 Jul: 90.

7. Dervilis N, Choi M, Taylor SG, Barthorpe RJ, Park G, Farrar CR, Worden K. On damage diagnosis for a wind turbine blade using pattern recognition. Journal of sound and vibration. 2014 Mar 17; 333(6):1833-1850.

8. Mollineaux M, Balafas K, Branner K, Nielsen P, Tesauro A, Kiremidjian A, Rajagopal R. Damage detection methods on wind turbine blade testing with wired and wireless accelerometer sensors. In EWSHM-7th European Workshop on Structural Health Monitoring 2014 Jul 8.

9. Shafiee M, Finkelstein M, Bérenguer C. An opportunistic condition-based maintenance policy for offshore wind turbine blades subjected to degradation and environmental shocks. Reliability Engineering \& System Safety. 2015 Oct 1; 142:463-471.

10. Slot HM, Gelinck ER, Rentrop C, van der Heide E. Leading edge erosion of coated wind turbine blades: Review of coating life models. Renewable Energy. 2015 Aug 1; 80:837-848. Kiil S. Erosion of wind turbine blade coatings-Design and analysis of jet-based laboratory equipment for performance evaluation. Progress in Organic Coatings. 2015 Jan 1; 78:103-115.
6. Godwin JL, Matthews P. Classification and detection of

11. Zhang S, Dam-Johansen K, Nørkjær S, Bernad Jr PL,

12. Li X, Yang Z, Zhang H, Du Z, Chen X. Crack growth sparse pursuit for wind turbine blade. Smart materials and structures. 2014 Nov 18; 24(1):015002.

13. Xie W, Zeng P, Lei L. A novel folding blade of wind turbine rotor for effective power control. Energy Conversion and Management. 2015 Sep 1; 101:52-65.

14. Hoell S, Omenzetter P. Structural damage detection in wind turbine blades based on time series representations of dynamic responses. In Smart Materials and Nondestructive Evaluation for Energy Systems 20152015 Mar 27 (Vol. 9439, p. 94390B). International Society for Optics and Photonics.

15. Wang YZ, Li F, Zhang X, Zhang WM. Composite wind turbine blade aerodynamic and structural integrated design optimization based on RBF Meta-Model. In Materials Science Forum 2015 (Vol. 813, pp. 10-18) Trans Tech Publications.

16. Haselbach PU, Bitsche RD, Branner K. The effect of delaminations on local buckling in wind turbine blades. Renewable Energy. 2016 Jan 1; 85:295-305.

17. Joshuva A, Sugumaran V. Fault diagnostic methods for wind turbine: A review. ARPN Journal of Engineering and Applied Sciences. 2016 Apr 11; 11(7):4654-4668.

18. Joshuva A, Sugumaran V. A data driven approach for condition monitoring of wind turbine blade using vibration signals through best-first tree algorithm and functional trees algorithm: A comparative study. ISA transactions. 2017 Mar 1; 67:160-172.

19. Joshuva A, Sugumaran V. Wind turbine blade fault diagnosis using vibration signals through decision tree algorithm. Indian Journal of Science and Technology. 2016 Dec 29; 9 (48):1-7.

20. Joshuva A, Sugumaran V, Amarnath M. Selecting kernel function of Support Vector Machine for fault diagnosis of roller bearings using sound signals through histogram features. International Journal of Applied Engineering Research. 2015; 10(68):482-487.

21. Joshuva A, Sugumaran V. A Comparative Study for Condition Monitoring on Wind Turbine Blade using Vibration Signals through Statistical Features: A Lazy Learning Approach. International Journal of Engineering and Technology. 2018; 7(4.10):190-196.

22. Joshuva A, Sugumaran V. A Machine Learning Approach For Condition Monitoring Of Wind Turbine Blade Using Autoregressive Moving Average (ARMA) Features Through Vibration Signals: A Comparative Study. Progress in Industrial Ecology-An International Journal. 2018; 12(1/2):14-34.

23. Joshuva A, Sugumaran V. Classification of various wind turbine blade faults through vibration signals using hyperpipes and voting feature intervals algorithm. International Journal of Performability Engineering. 2017; 13(3):247-258.

24. Joshuva A, Sugumaran V. Failure Analysis on Wind Blade Using Vibration Signals and Classifying the Failures Using Logit Boost Algorithm. International Journal of Control Theory and Applications. 2016; 9(52):225-234.

25. Joshuva A, Sugumaran V. A comparative study of Bayes classifiers for blade fault diagnosis in wind turbines through vibration signals. Struct Durab Health Monit (SDHM). 2017; 12(1):69-90.

26. More Vasudha, Panditrao Harshal, Joshuva A, Sugumaran V. Effect of Sampling Frequency and Sample Length on Fault Diagnosis of Wind Turbine Blade. Pakistan Journal of Biotechnology. 2018; 15(Special 
Issue ICRAME 17):14-17.

27. Shoombuatong W, Hongjaisee S, Barin F, Chaijaruwanich J, Samleerat T. HIV-1 CRF01_AE coreceptor usage prediction using kernel methods based logistic model trees. Computers in biology and medicine. 2012 Sep 1; 42(9):885-889.

28. Joshuva A, Sugumaran V. Fault Diagnosis of Wind Turbine Blade Using Vibration Signals through J48 Decision Tree Algorithm and Random Tree Classifier. International Journal of Control Theory and Applications. 2016; 9(52):249-258.

29. Joshuva A, Sugumaran V. Multiclass Classifier Approach for Fault Diagnosis of Wind Turbine Blade Using Vibration Signals through Statistical Analysis. International Journal of Control Theory and Applications. 2016; 9(52):235-247.

30. Joshuva A, Sugumaran V, Amarnath M, Lee SK. Remaining Life-Time Assessment of Gear Box Using Regression Model. Indian Journal of Science and Technology. 2016 Dec 28; 9(47):1-8.

31. Joshuva A, Sugumaran V. A Study of Various Blade Fault Conditions on a Wind Turbine Using Vibration Signals through Histogram Features. Journal of Engineering Science and Technology. 2018 Jan; 13(1):102-121.

32. Manju BR, Joshuva A, Sugumaran V. A Data Mining Study for Condition Monitoring on Wind Turbine Blades Using Hoeffding Tree Algorithm Through Statistical and Histogram Features. International Journal of Mechanical Engineering and Technology. 2018; 9(1):1061-1079.

33. Joshuva A, Sugumaran V. Wind Turbine Blade Fault Diagnosis Using Vibration Signals and Statistical Features through Nested Dichotomy Classifiers. International Journal of Pharmacy and Technology. 2017 Apr; 9(1): 29147-29164.

34. Joshuva A, Sugumaran V. Fault Diagnosis for Wind Turbine Blade through Vibration Signals Using Statistical Features and Random Forest Algorithm. International Journal of Pharmacy and Technology. 2017 Apr; 9(1): 28684-28696.

35. Deenadayalan, Sivakumar, S., Vishnuvardhan R., Sathish Kumar R. Fabrication and Characterisation of B-H-G Fiber with Teak Wood Particles Reinforced Hybrid Composite. International Journal of Engineering and Technology. 2018; 7(2.31): 208-211.

36. Sivakumar S, Dhanalakshmi V, Vishuvardhan R. Extraction of Subtractive Features of Prismatic Parts from STEP File for CAD/CAM Integration. Journal of Science and Technology, Malaysia. 2019 Jan; 27 (1): 343-356.

37. Vishnuvardhan R, Pooranam N, Sivakumar S, Vignesh T. Producing Electrical Energy from Light Intensity, Design Traking System by Dual Axis Method. International Journal of Mechanical and Production Engineering Research and Development (IJMPERD). 2019; Jan; 9: 503-509.

38. Joshuva A, Sugumaran V. Improvement in wind energy production through condition monitoring of wind turbine blades using vibration signatures and ARMA features: a data-driven approach. Progress in Industrial Ecology, an International Journal. 2019 Jun 21; 13(3):207-31.

39. Joshuva A, Sugumaran V. Selection of a meta classifierdata model for classifying wind turbine blade fault conditions using histogram features and vibration signals: a data-mining study. Progress in Industrial Ecology, an International Journal. 2019 Jun 21; 13(3):232-51

40. Joshuva A, Sugumaran V. Crack Detection and Localization on Wind Turbine Blade Using Machine Learning Algorithms: A Data Mining Approach. Struct Durab Health Monit (SDHM). 2019; 13(2):181-203.
41. Joshuva A, Sugumaran V. Fault Diagnosis and Localization of Wind Turbine Blade [dissertation], Vellore Institute of Technology, Chennai Campus. 2018. 